\title{
Approximate solution of the system of nonlinear integral equation by Newton- Kantorovich method
}

\begin{abstract}
The Newton-Kantorovich method is developed for solving the system of nonlinear integral equations. The existence and uniqueness of the solution are proved, and the rate of convergence of the approximate solution is established. Finally, numerical examples are provided to show the validity and the efficiency of the method presented.
\end{abstract}

Keyword: Newton-Kantorovich method; Rate of convergence; Nonlinear operator; Volterra integral equation; Trapezoidal formula 